EPJ Web of Conferences 71, 00015 (2014)

DOI: 10.1051/epjconf/20147100015

(C) Owned by the authors, published by EDP Sciences, 2014

\title{
Hard Probes at RHIC
}

\author{
Jana Bielčíková ${ }^{1, a}$ \\ ${ }^{1}$ Nuclear Physics Institute ASCR, Na Truhlářce 39/64, 18086 Prague, Czech Republic
}

\begin{abstract}
Measurements of jets and heavy flavour, the so called hard probes, play a crucial role in understanding properties of hot and dense nuclear matter created in high energy heavy-ion collisions. The measurements at the Relativistic Heavy Ion Collider (RHIC) showed that in central Au+Au collisons at RHIC energy $\left(\sqrt{s_{N N}}=200 \mathrm{GeV}\right)$ the nuclear matter created has properties close to those of perfect liquid, manifests partonic degrees of freedom and is opaque to hard probes. In order to draw quantitative conclusions on properties of this hot and dense nuclear matter reference measurements in proton-proton collisions and $\mathrm{d}+\mathrm{Au}$ collisions are essential to estimate cold nuclear matter effects. In this proceedings a review of recent results on hard probes measurements in $\mathrm{p}+\mathrm{p}, \mathrm{d}+\mathrm{Au}$ and $\mathrm{A}+\mathrm{A}$ collisions as well as of beam energy dependence of jet quenching from STAR and PHENIX experiments at RHIC is presented.
\end{abstract}

\section{Introduction}

Studies of nuclear matter under extreme conditions of energy density and temperature are on the frontiers of research carried in modern nuclear physics. The scope of this research goes however far beyond the field of nuclear physics and plays also an important role in astrophysics and particle physics. The Relativistic Heavy Ion Collider (RHIC) at Brookhaven National Laboratory is currently the only facility in the world dedicated to systematic studies of nuclear matter under extreme conditions of high energy density and temperature. The calculations from lattice QCD demonstrate that at energy densities of about $1 \mathrm{GeV} / \mathrm{fm}^{3}$ and at temperatures of about $170 \mathrm{MeV}$, that are exceeded in nuclear collisions at RHIC, the phase transition to a new state of matter, the Quark Gluon Plasma (QGP), where quarks and gluons are deconfined, should take place.

The observation and exploration of QGP is an experimentally challenging task because of its elusive characteristics. Combining multiple experimental signatures, all four RHIC experiments in 2005 concluded [1-4] that an unprecedented state of nuclear matter demonstrating very strong collective flow effects at parton level and behaving as a strongly-coupled liquid (sQGP) with a very low ratio of shear viscosity to entropy was created in central $\mathrm{Au}+\mathrm{Au}$ collisions at the center of mass energy $\sqrt{s_{N N}}=200 \mathrm{GeV}$ per nucleon-nucleon pair. This finding was in contrast with theoretical predictions in which a weakly-coupled gas of quarks and gluons was expected to be formed. The matter created at RHIC is opaque to energetic quarks and gluons resulting in large suppression of particle production at large transverse momentum $\left(p_{T}\right)$ relative to $\mathrm{p}+\mathrm{p}$ collisions or $\mathrm{d}+\mathrm{Au}$ collisions, where only cold

a e-mail: jana.bielcikova@ujf.cas.cz 
nuclear matter effects are expected to be present. This so called "jet quenching" effect is observed for light $(u, d, s)$ as well as heavy ( $c$ and $b)$ quark flavours.

In this proceedings, an overview of recent results on measurements of beam energy dependence of jet quenching and measurements of jets and heavy flavour, the so called hard probes, at RHIC is given. Hard probes play an essential role in understanding properties of hot and dense nuclear matter created in high energy heavy ion collisions because they are created early in the collision. Production of hard probes can be influenced by two types of nuclear effects: cold nuclear matter effects and/or final state effects due to parton interactions with the produced nuclear matter. For complete understanding of existing data measurements in various collision systems are necessary, where RHIC and its flexibility of a large variety of collision species from deuteron up to uranium including asymmetric collisions plays essential role.

\section{Energy dependence of jet quenching}

One of the observables associated with particle production in hot and dense nuclear matter is the nuclear modification factor defined as the ratio of the transverse momentum spectrum in central $\mathrm{A}+\mathrm{A}$ collisions relative to that in $\mathrm{p}+\mathrm{p}$ collisions scaled by corresponding number of binary collisions, $N_{\text {coll }}$, calculated in Monte Carlo Glauber model [5]:

$$
R_{A A}=\frac{1}{N_{\text {coll }}^{\text {central }}} \frac{\left(\frac{d^{2} N}{d p_{T} d \eta}\right)^{\text {central }}}{\left(\frac{d^{2} N}{d p_{T} d \eta}\right)^{p+p}}
$$

Already the very first measurements at RHIC showed that particle production is strongly suppressed in central $\mathrm{Au}+\mathrm{Au}$ collisions relative to $\mathrm{p}+\mathrm{p}$ collisions [1-4]. For charged particles the suppression reaches factor of about 4-5 and the suppression is shown to persists out to the largest transverse momenta currently allowed by the statistics of available data as demonstrated in Figure 1. Besides $R_{A A}$ of charged particles, in Figure 1 the nuclear modification factor of direct photons is presented as well. As photons do not interact strongly, their nuclear modification factor is consistent with unity.

In order to explore the QCD phase diagram in detail, a first phase of a dedicated Beam Energy Scan (BES) program at RHIC took place in 2010-2011. Both STAR and PHENIX experiments have measured $\mathrm{Au}+\mathrm{Au}$ collisions at $\sqrt{s_{N N}}=7.7,11.5,19.6,27,39$, and $62.4 \mathrm{GeV}$ with an aim to search for a critical point and a phase boundary marked by the disappearance of key signatures of quark gluon plasma. As currently no measurements of $\mathrm{p}+\mathrm{p}$ data at these collision energies (except of $\sqrt{s_{N N}}=62.4$ $\mathrm{GeV}$ ) exist, the peripheral $\mathrm{Au}+\mathrm{Au}$ collisions are used instead of $\mathrm{p}+\mathrm{p}$ collisions and the central-toperipheral ratio scaled in analogy with $R_{A A}$ by its respective average number of $N_{\text {coll }}$ was evaluated:

$$
R_{C P}=\frac{N_{\text {coll }}^{\text {peripheral }}}{N_{\text {coll }}^{\text {central }}} \frac{\left(\frac{d^{2} N}{d p_{T} d \eta}\right)^{\text {central }}}{\left(\frac{d^{2} N}{d p_{T} d \eta}\right)^{\text {peripheral }}}
$$

Please note that peripheral $\mathrm{Au}+\mathrm{Au}$ collisions are not exactly the same as $\mathrm{p}+\mathrm{p}$ collisions due to presence of cold nuclear matter $(\mathrm{CNM})$ effects. Figure 2 shows the transverse momentum dependence of the nuclear modification factor $R_{C P}$ measured by the STAR experiment for different collision energies $[6,7]$. For large $p_{T}$, a smooth transition from suppression to enhancement is observed around $\sqrt{s_{N N}}=39 \mathrm{GeV}$. Quenching of particle production in hot and dense nuclear matter competes with the Cronin effect or radial boost, which in turn cause enhancement of particle production in nuclear matter relative to elementary collisions. The relative contributions from these processes and jet quenching are currently under investigation. In [6, 7], data are compared with simulations using HIJING (Heavy 


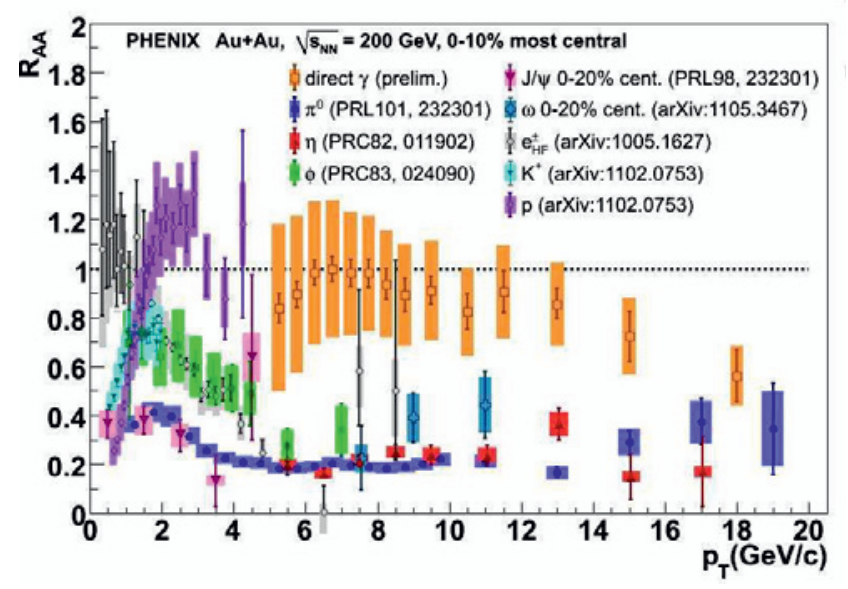

Figure 1. Collection of measured nuclear modification factors by the PHENIX experiment for various particle species (see legend) in $\mathrm{Au}+\mathrm{Au}$ collisions at $\sqrt{s_{N N}}=200 \mathrm{GeV}$.

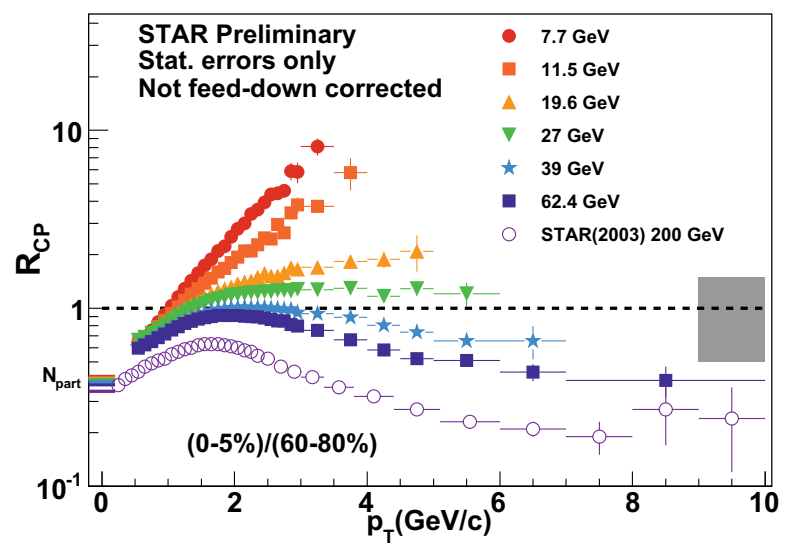

Figure 2. Charged hadron $R_{C P}$ measured by the STAR experiment during the RHIC Beam Energy Scan program. The gray error band shows combined systematic uncertainty (added in quadrature) on number of binary collisions scaling and $20 \%$ deviation between the published STAR data (feeddown corected) and new data (without feeddown correction). The dashed line at 1 indicates the $N_{\text {coll }}$ scaling, while different $N_{\text {part }}$ colored lines correspond to $N_{\text {part }}$ production scaling for individual measured values of $\sqrt{s_{N N}}$. The figure is taken from [6].

Ion Jet INteraction Generator) [8] and AMPT (A Multi-Phase Transport) [9] models. Neither of these two models is able to reproduce the data in its complexity. Further investigations of nuclear modification factors exploring particle production in asymmetric collisions of light and heavy nuclei are expected to provide additional constraints on models. 


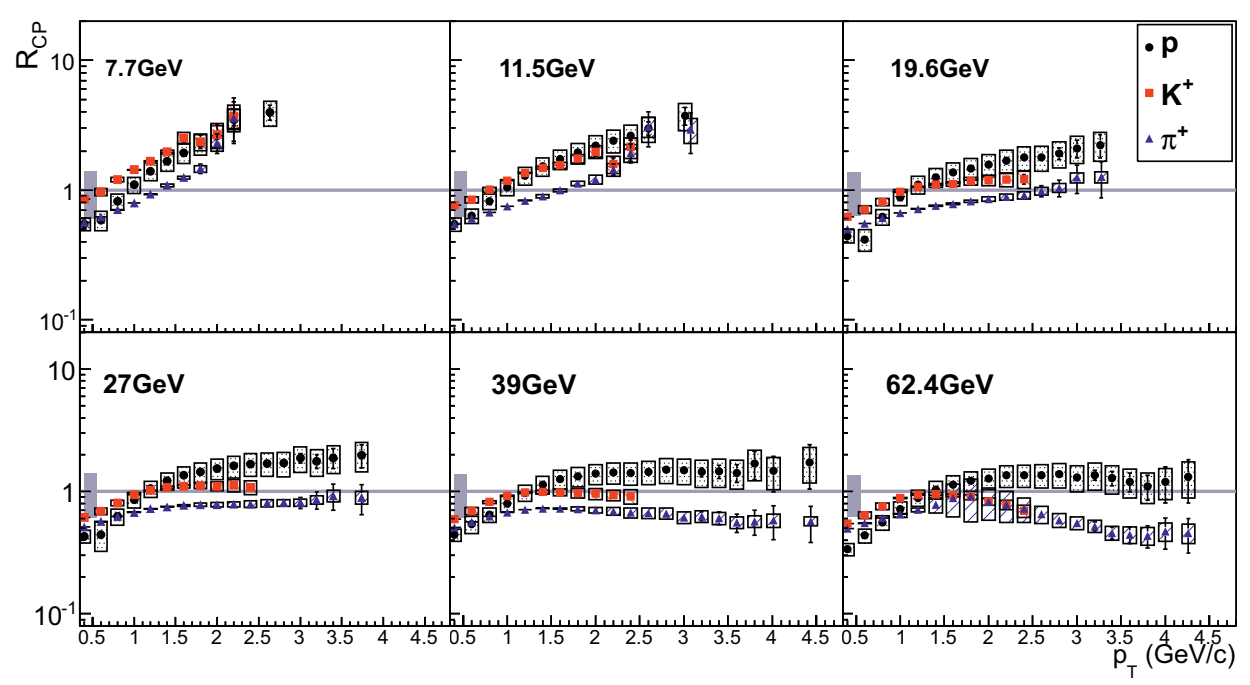

Figure 3. (The nuclear modification factor $R_{\mathrm{CP}}$ for identified $p, K^{+}$, and $\pi^{+}$for RHIC BES energies measured by STAR. The boxes are $p_{\mathrm{T}}$ dependent systematic uncertainties due to particle identification while the $p_{\mathrm{T}}$ independent uncertainty is from $N_{\text {coll }}$ scaling. The figure is taken from [10].

Based on information from ionization energy loss in Time Projection Chamber (TPC) and from the Time Of Flight (TOF) detector, the STAR experiment was also able to perform measurements of $R_{C P}$ for protons, pions and charged kaons separately. The preliminary results [10] for individual collision energies are displayed in Figure 3. In $p_{T}$ range from about 2.5 to $4 \mathrm{GeV} / c$ protons are not suppressed at any beam energy from $\sqrt{s_{N N}}=7$ to $62.4 \mathrm{GeV}$. In contrast, pion production is enhanced only at lower beam energies with a transition point near $\sqrt{s_{\mathrm{NN}}}=27 \mathrm{GeV}$ and at higher collision energies the pion production is strongly suppressed. This corroborates and extends earlier measurements at the top RHIC energy [11], which showed that pions suffer larger suppression than protons. In the momentum range where particle identification is feasible, pions could be therefore used as a better probe to study onset of jet quenching.

\section{Charm production}

Particles containing charm $(c)$ or beauty $(b)$ quarks, the so called heavy flavours, provide due to their large mass higher sensitivity to medium properties in comparison with light quark flavours. However the rate of heavy flavour production is much smaller and thus large data statistics is needed. In last years, the increased luminosity of RHIC and data taking rates allowed to significantly improve statistics and start to investigate in a systematic way charm production. The charm production can be indirectly measured via heavy flavour electrons, also commonly referred to as "non-photonic electrons" (NPE), which are dominantly originating from $c$ and $b$ decays or directly via hadronic decays of $D$ mesons.

For measurements in heavy-ion collisions it is first necessary to understand heavy flavour production in elementary collision systems such as $\mathrm{p}+\mathrm{p}$. Both STAR and PHENIX experiments have measured NPE inclusive transverse momentum spectra in $p+p$ collisions at top RHIC energy [15-17]. 


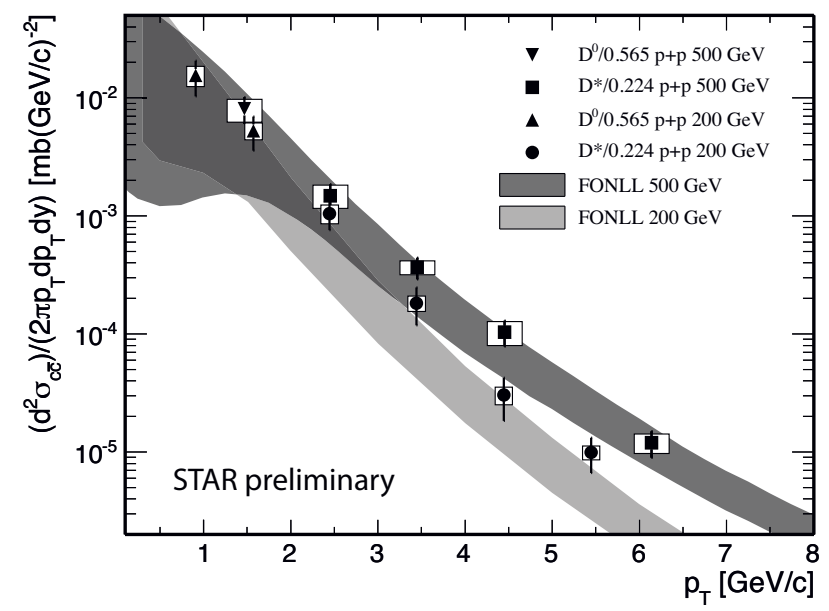

Figure 4. Transverse momentum dependence of $c \bar{c}$ production cross section calculated from $D^{0}$ and $D^{*}$ yields in $\mathrm{p}+\mathrm{p}$ collisions at $\sqrt{s}=200 \mathrm{GeV}[12]$ and $500 \mathrm{GeV}$ [13] measured by the STAR experiment. The data are compared to FONLL calculations [14].

Here we feature recent STAR measurements of $c \bar{c}$ production cross section from hadronic decays of $D^{0}$ and $\mathrm{D}^{*}$ mesons in $\mathrm{p}+\mathrm{p}$ collisions at $\sqrt{s_{N N}}=200 \mathrm{GeV}$ [12] and $500 \mathrm{GeV}$ [13]. The charm spectra at both collision energies are shown in Figure 4 and are compared to the Fixed-Order-Next-to-LeadingLogarithm (FONLL) calculations from [14]. The FONLL calculations are found to agree well with data given current experimental and theoretical uncertainties.

It was originally expected that in the medium heavy quarks will exhibit less suppression than light quarks, due to a suppression of gluon radiation at small angles known as the "dead cone" effect [18]. However, already the early measurements in central $\mathrm{Au}+\mathrm{Au}$ collisions at RHIC revealed that production of heavy flavour electrons show surprisingly similar level of suppression as that of light quarks [19-21]. Several mechanisms have been then suggested to explain the large heavy flavour electron suppression in addition to radiative energy loss. Models, which incorporate also collisional energy loss and energy loss due to in-medium dissociation of heavy flavour D and B mesons, demonstrated to be more successful in description of heavy flavour electron $R_{A A}$, see e.g. [22-24].

Here we demonstrate the large heavy flavour production suppression on the newest STAR data [13, 25]. The left panel of Figure 5 shows the nuclear modification factor for heavy flavour electrons and $D^{0}$ mesons in $0-10 \%$ most central $\mathrm{Au}+\mathrm{Au}$ collisions at $\sqrt{s_{N N}}=200 \mathrm{GeV}$. As it can be seen, the suppression of heavy flavour electrons and charm mesons is consistent with each other and with that of pions. In the right panel of Figure 5 a closer investigation of centrality dependence of the nuclear modification factor of $D^{0}$ mesons in central as well as minimum bias $\mathrm{Au}+\mathrm{Au}$ collisions in the transverse momentum range $0<p_{T}<6 \mathrm{GeV} / c$ is displayed. The data show a peaked structure with a maximum around $p_{T}=1-3 \mathrm{GeV} / c$. At higher momenta $\left(p_{T}>3 \mathrm{GeV} / c\right)$, there is a strong suppression of $D^{0}$ yields which increases with centrality. This indicates large energy loss of charm quarks traversing through the medium. The STAR results are also compared with two transport model calculations $[26,27]$ based on the relativistic Langevin simulation. The calculations describe well the shape of the nuclear modification factor and interpret the maximum as being caused by the transverse 

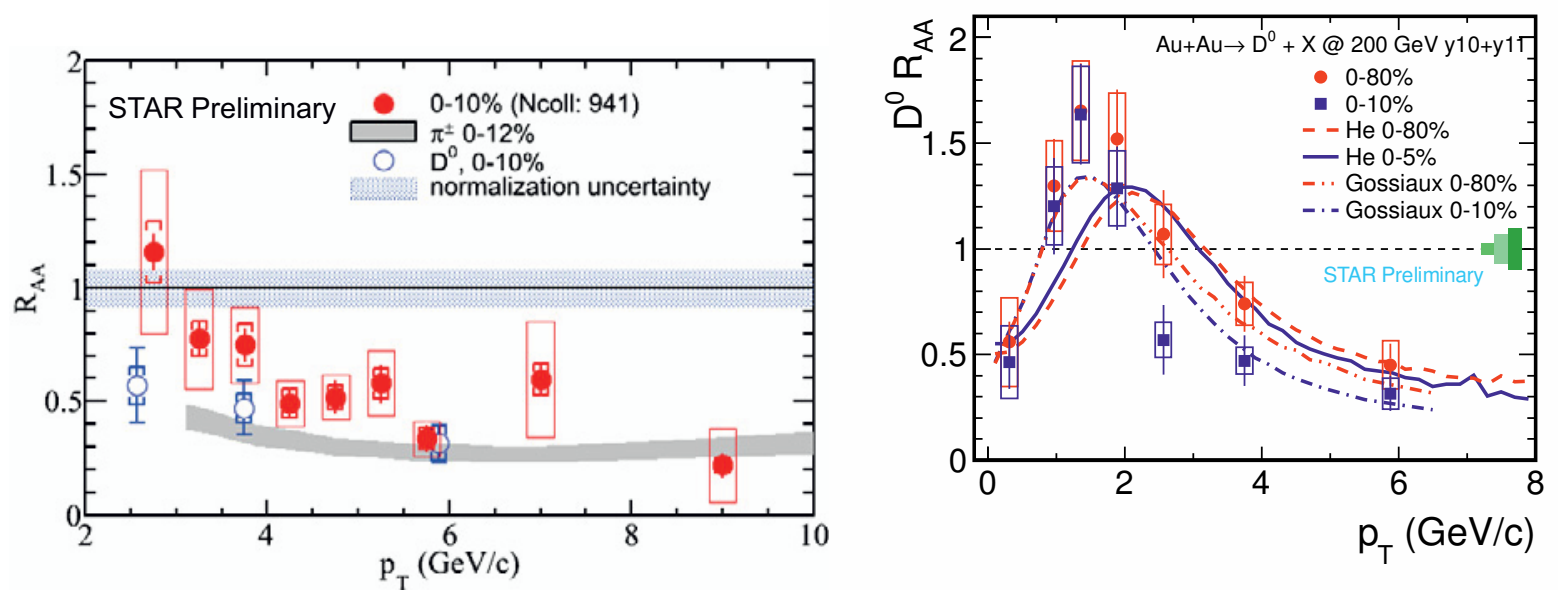

Figure 5. (Left) Transverse momentum dependence of nuclear modification factor of non-photonic electrons (full circles) and $D^{0}$ mesons (open circles) in $0-10 \%$ most central $\mathrm{Au}+\mathrm{Au}$ collisions at $\sqrt{s_{N N}}=200 \mathrm{GeV}$ from the STAR experiment. For comparison the nuclear modification factor of pions is shown as well (grey band). (Right) Nuclear modification factor of $D^{0}$ meson vs $p_{T}$ for $0-10 \%$ and $0-80 \%$ centralities in $\mathrm{Au}+\mathrm{Au}$ collisions at $\sqrt{s_{N N}}=200 \mathrm{GeV}$ measured by the STAR experiment. The data are compared to two transport model calculations (see legend). Error bars on the data points are statistical while boxes represent systematic uncertainties. The figures are taken from [13].

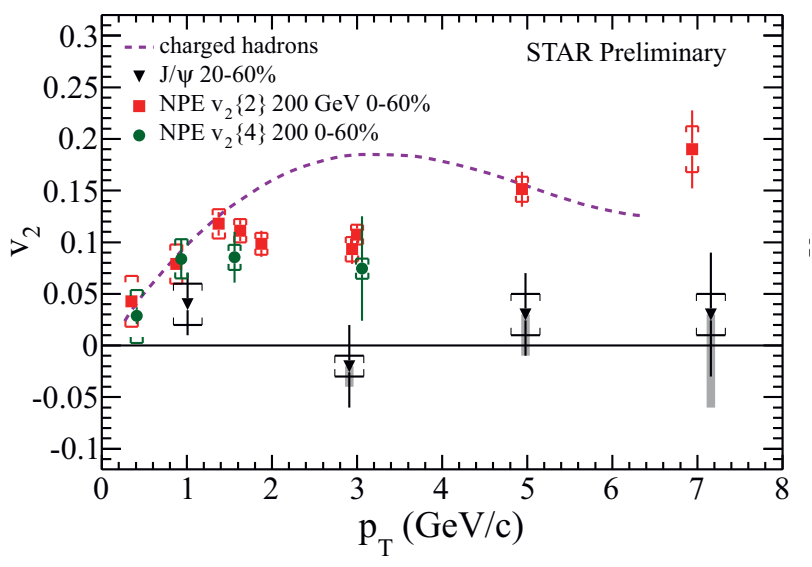

Figure 6. Measurement of heavy flavour electron (NPE) elliptic flow $v_{2}$ as a function of $p_{T}$ in $0-60 \% \mathrm{Au}+\mathrm{Au}$ collisions at $\sqrt{s_{N N}}=200 \mathrm{GeV}$. For comparison, the charged hadron (dashed line) and $\mathrm{J} / \psi$ (closed triangle down) elliptic flow is also shown. The figure is taken from [25]. 


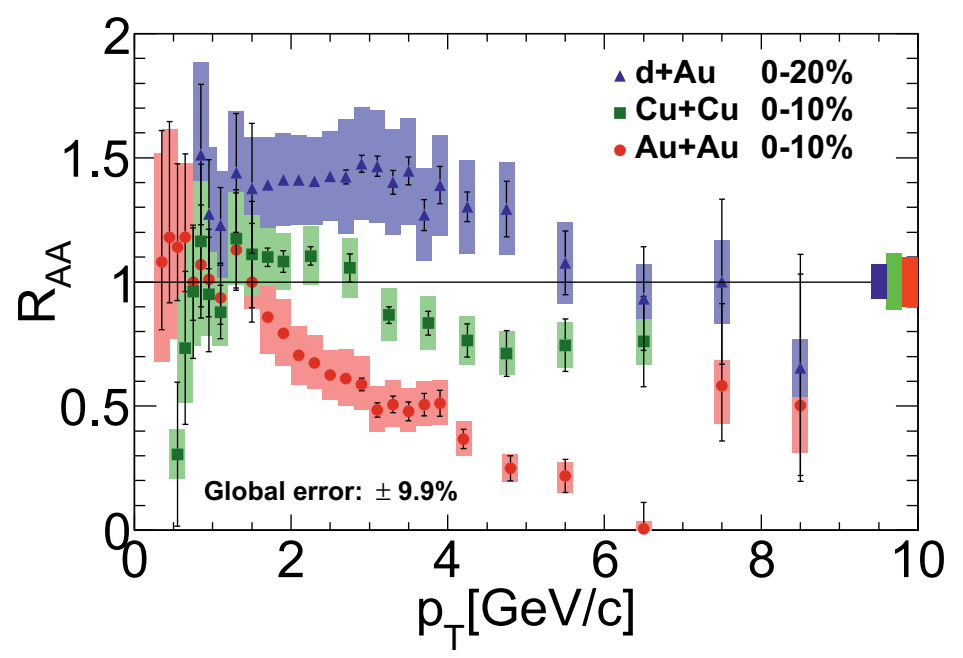

Figure 7. The nuclear modification factor of heavy flavour electrons at midrapidity in central $\mathrm{d}+\mathrm{Au}, \mathrm{Cu}+\mathrm{Cu}$, and $\mathrm{Au}+\mathrm{Au}$ collisions at $\sqrt{s_{N N}}=200 \mathrm{GeV}$ from the PHENIX experiment [28]. The boxes around data points correspond to systematic uncertainties, which include the $N_{\text {coll }}$ scaling error. The global uncertainty is that of the $\mathrm{p}+\mathrm{p}$ reference data.

flow picked up from the expanding medium. In addition, a large anisotropic elliptic flow $\left(v_{2}\right)$ of heavy flavour electrons have been observed in $\mathrm{Au}+\mathrm{Au}$ colisions at $\sqrt{s_{N N}}=200 \mathrm{GeV}$ as demonstrated in Figure 6 . These $v_{2}$ values are systematically higher than those measured at lower collision energies $\sqrt{s_{N N}}=39$ and $62.4 \mathrm{GeV}$, respectively [25]. The large $v_{2}$ values provide additional indication of strong interaction of charm quarks with the medium and pose a challenge for theoretical calculations to describe simultaneously both $R_{A A}$ and $v_{2}$.

The PHENIX experiment has recently investigated the system size dependence of heavy flavour electron production [28]. The data (cf. Figure 7) show a clear enhancement in central $d+$ Au collisions, a slight suppression in central $\mathrm{Cu}+\mathrm{Cu}$ collisions, and the large suppression of heavy flavour electrons in the most central $\mathrm{Au}+\mathrm{Au}$ collisions as already discussed above. A closer investigation of $R_{A A}$ of nonphotonic electrons in various collision systems is shown in Figure 8, where the average $R_{A A}$ values in two different $p_{T}$ ranges $\left(1<p_{T}<3 \mathrm{GeV} / c\right.$ and $\left.3<p_{T}<5 \mathrm{GeV} / c\right)$ are displayed as a function of $N_{\text {coll }}$. The data manifest a common trend of increasing enhancement followed by suppression at large $N_{\text {coll }}$ values. The presence of an enhancement in peripheral $\mathrm{Cu}+\mathrm{Cu}$ collisions and $\mathrm{d}+\mathrm{Au}$ collisions at similar values of $N_{\text {coll }}$ as well as $N_{\text {part }}$ (not shown here) implies that already in the initial state of heavyion collisions there exist effects influencing heavy-quark production. Central $\mathrm{Cu}+\mathrm{Cu}$ collisions show similar level of suppression as semi-peripheral $\mathrm{Au}+\mathrm{Au}$ collisions of a similar system size indicating increasing importance of hot nuclear matter.

It is interesting to confront the enhancement of heavy flavour electron production in $\mathrm{d}+\mathrm{Au}$ and peripheral $\mathrm{Cu}+\mathrm{Cu}$ collisions with the observed mass dependent enhancement of pions, kaons and protons [29]. In general, $R_{A A}$ values larger than unity are commonly attributed to the "Cronin effect" [30]. The original physics mechanism behind the Cronin effect relies on $k_{t}$ boosts to partons via multiple scattering in the nucleus before the hard scattering and fragmentation and cannot therefore explain the 

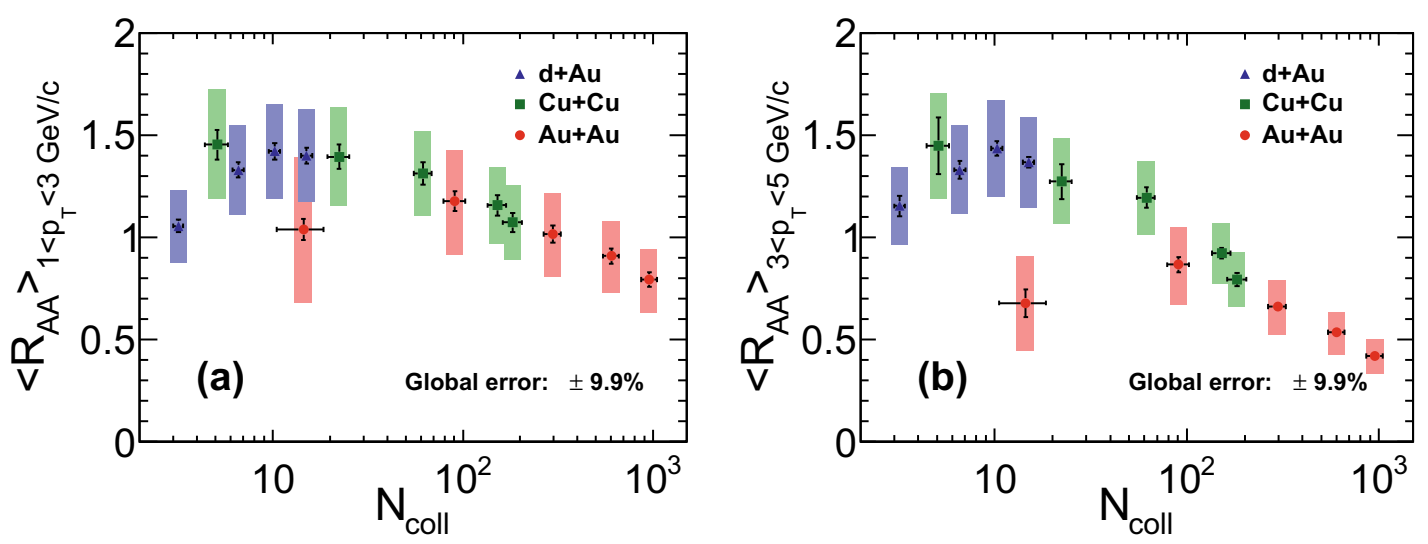

Figure 8. The nuclear modification factor $R_{A A}$ of heavy flavour electrons at midrapidity in $\mathrm{d}+\mathrm{Au}, \mathrm{Cu}+\mathrm{Cu}$ and $\mathrm{Au}+\mathrm{Au}$ collisions at $\sqrt{s_{N N}}=200 \mathrm{GeV}$ from the PHENIX experiment. The $R_{A A}$ values are plotted as a function of $N_{\text {coll }}$ in two different $p_{T}$ ranges: $1<p_{T}<3 \mathrm{GeV} / c$ (left) and $3<p_{T}<5 \mathrm{GeV} / c$ (right) [28].

observed particle mass ordering. Further theoretical studies including parton recombination as well as future measurements of charmed baryons and mesons in cold nuclear matter are expected to bring more insights.

\section{Properties of fully reconstructed jets}

Measurements of nuclear modification of leading hadrons with large $p_{T}$ are known to suffer from several limitations. The leading hadrons are a mixture of parent quarks and gluons and in addition as a result of fragmentation, they carry only a fraction of energy of its parent parton. Thus they ultimately sample a wide range of parton energies. In addition, the leading hadrons once measured are more likely to come from the surface of the expanding medium [31]. Fully reconstructed jets overcome to large extent these limitations, however their reconstruction in nucleus-nucleus collisions is difficult because of their relatively small production rate and large fluctuating background. The pioneering work in full jet reconstruction was carried out by the STAR experiment in 2008 and 2009 [32-35]. The approach chosen by the STAR experiment is based on modern sequential recombination jet reconstruction algorithms anti- $k_{T}$ and $k_{T}$ and associated background correction procedures available in the FastJet package [36]. The jet reconstruction algorithms were used in the massless recombination scheme and the jet reconstruction was performed for two resolution parameters $R=0.2$ and $R=0.4$, respectively. Fragmentation biases were minimized by using as low as possible cuts on charged track $p_{T}$ and tower energy in the STAR electromagnetic calorimeter (BEMC) of $0.2 \mathrm{dGeV}$. To avoid double counting, BEMC towers matched with one or more TPC tracks were removed from analysis. The background is estimated using the $k_{T}$ algorithm and background subtraction is applied event-wise according to

$$
p_{T}^{\text {rec }}=p_{T}^{\text {candidate }}-\rho \cdot A,
$$

where $\rho$ corresponds to a $p_{T}$-weighted background density in an event and $A$ to a jet area. For central $\mathrm{Au}+\mathrm{Au}$ collisions at top RHIC energy, the average background density at midrapidity reaches about 

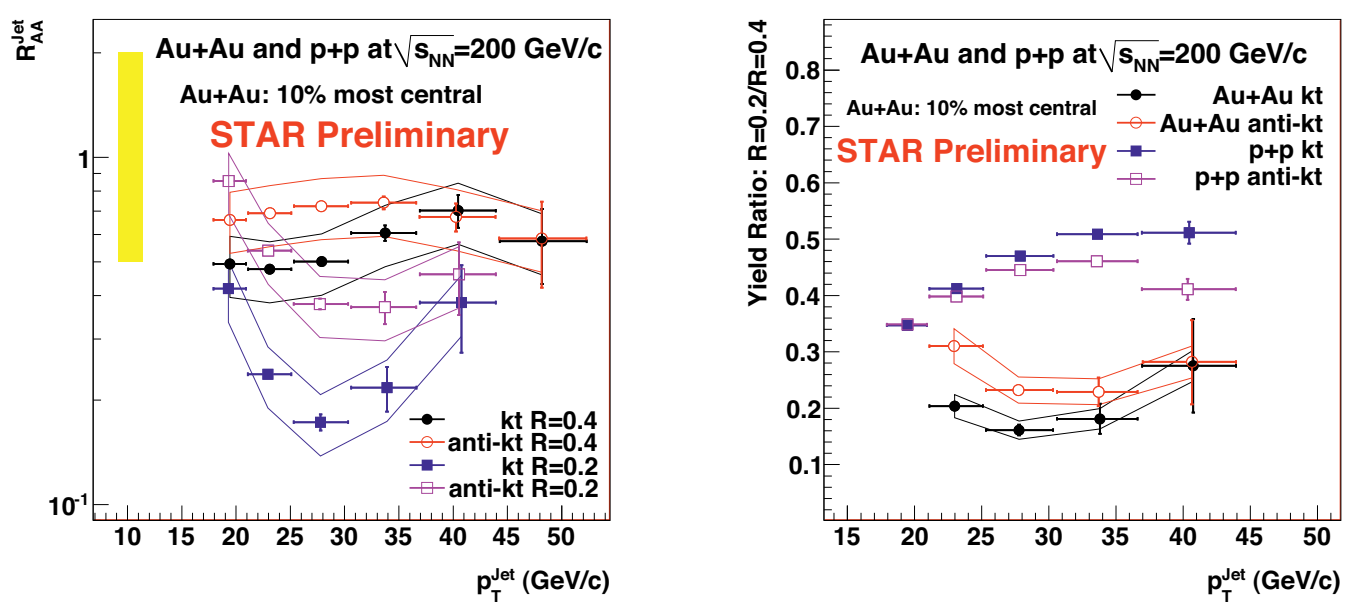

Figure 9. (Left) Nuclear modification factor of inclusive jets in Au+Au collisions measured by the STAR experiment at $\sqrt{s_{N N}}=200 \mathrm{GeV}$. The jets were reconstructed with $k_{T}$ and anti- $k_{T}$ recombination algorithms with the resolution parameter $R=0.2$ and 0.4. (Right) The ratio of inclusive jet cross sections for $R=0.2$ and $R=0.4$ at the same collision energy. The figures are taken from [34].

$75 \mathrm{GeV}$ per unit area. In these early analyses, fluctuations around the avaraged background value were approximated by a Gaussian distribution. Prior to the unfolding procedure which takes care of background fluctuations and detector effects, the signal jet spectrum was corrected for "false"-jet yield, defined as the signal in excess of the background model from random association of uncorrelated soft particles. The false jet yield was estimated by reconstructed jets in randomized $\mathrm{Au}+\mathrm{Au}$ collisions, with leading jets removed.

Figure 9 (left panel) shows the nuclear modification of jet yield in central $\mathrm{Au}+\mathrm{Au}$ collisions at $\sqrt{s_{N N}}=200 \mathrm{GeV}$ relative to that in $\mathrm{p}+\mathrm{p}$ collisions scaled by the respective $N_{\text {coll }}$. The systematic uncertainty on the jet energy scale is shown as the vertical shaded band and is dominated by the uncertainties on the BEMC calibration (5\%), unobserved jet energy (3\%), and charged component momentum resolution (2\%). Solid lines represent systematic uncertainty on the jet yield in $\mathrm{Au}+\mathrm{Au}$ collisions due to background fluctuations. These pioneering measurements found the jet $R_{A A}$ for $R=0.4$ within the large statistical and systematic uncertainties to be compatible with unity, expected in case of unbiased jet reconstruction. The $R_{A A}$ of jets with the resolution parameter $R=0.2$ was found to be systematically below that for $R=0.4$. The significant differences between $k_{T}$ and anti- $k_{T}$ algorithms can be attributed to their different response to presence of large background. The anti$k_{T}$ algorithm is less susceptible to presence of the background because the clustering starts from constituents with large $p_{T}$, in contrast to the $k_{T}$ algorithm which clusters first together consitutents of low $p_{T}$. Therefore nowadays typically only results from the anti- $k_{T}$ algorithm are reported.

The right panel of Figure 9 displays the ratio of jet yield for $R=0.2$ over that for $R=0.4$ for $\mathrm{p}+\mathrm{p}$ and $\mathrm{Au}+\mathrm{Au}$ collisions. In this observable the large jet energy scale systematic uncertainties cancel. In case of $\mathrm{p}+\mathrm{p}$ collisions the ratio increases with jet $p_{T}$ and is consistent with a PYTHIA calculation but not with NLO QCD calculation [37]. The same ratio is strongly suppressed for central $\mathrm{Au}+\mathrm{Au}$ relative to $p+p$ collisions, pointing to broadening of jets in the nuclear matter. Interested reader is 

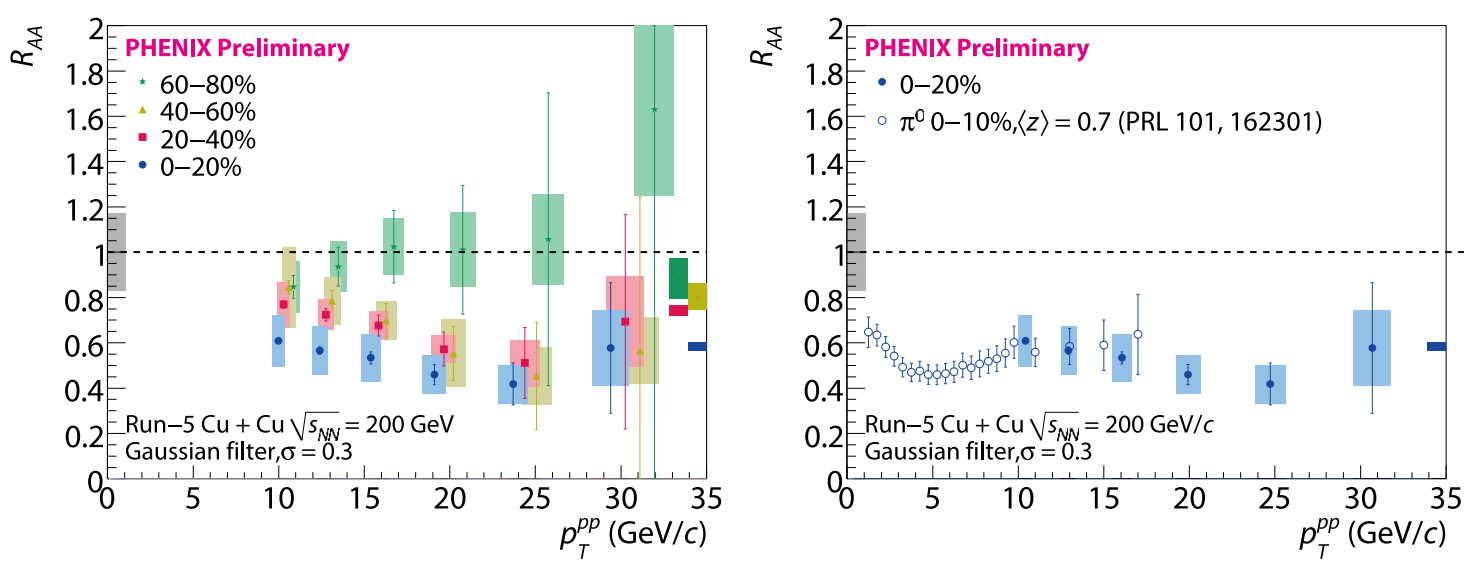

Figure 10. (Left) Nuclear modification factor of jets in $\mathrm{Cu}+\mathrm{Cu}$ collisions at $\sqrt{s_{N N}}=200 \mathrm{GeV}$ measured by the PHENIX experiment in Run 5. (Right) Comparison between the jet and $\pi^{0}$ nuclear modification factor in central $\mathrm{Cu}+\mathrm{Cu}$ collisions at the same collision energy. The shaded box to the left in both figures indicates the systematic uncertainty in the jet energy scale, shaded boxes to the right show centrality dependent systematic uncertainty between embedding and unfolding, shaded boxes associated with data points indicate point-to-point systematic uncertainties. The figures are taken from [41].

referred to [38], where a NLO QCD calculation which addresses these measurements was carried out. In addition, we would like to point to recent developments in full jet reconstruction in STAR which were shown after this conference at the Hard Probes 2013 conference, where new high statistics data and most up to date analysis techniques used also by the ALICE experiment at LHC were shown [39].

Contrary to the STAR experiment, the PHENIX experiment has only limited acceptance in azimuth and therefore uses a cone reconstruction algorithm based on Gaussian filtering developed in [40]. This algorithm takes advantage of the collimated emission of hadrons and is insensitive to large angle fragments.

In the left panel of Figure 10 the extracted jet nuclear modification factor $R_{A A}$ is shown after correction for detector effects [41-43]. The shaded box to the left indicates the systematic uncertainty in the jet energy scale, shaded boxes to the right show centrality dependent systematic uncertainty between embedding and unfolding and shaded boxes associated with data points correspond to pointto-point systematic uncertainties. In the right panel of Figure 10, the jet $R_{A A}$ is compared with the nuclear modification factor of neutral pions in $20 \%$ most central $\mathrm{Cu}+\mathrm{Cu}$ collisions [44]. Although the $R_{A A}$ of $\pi^{0} \mathrm{~s}$ has a different energy scale than fully reconstructed jets, both nuclear modification factors manifest approximately flat behaviour within the accesssible transverse momentum range. The suppression of jet $R_{A A}$ is increasing with $\mathrm{Cu}+\mathrm{Cu}$ collision centrality and in the $20 \%$ most central collisions reaches values of about $0.5-0.6$, which are compatible with that of $\pi^{0}$.

It is also important to study the di-jet azimuthal correlation shown in Figure 11 to look for possible de-correlation caused by the medium. The Gaussian widths extracted from the fit to corrected azimuthal distribution in the range $7.5 \mathrm{GeV} / c<p_{T}<11.5 \mathrm{GeV} / c$ were found to be within uncertainties independent of centrality. 


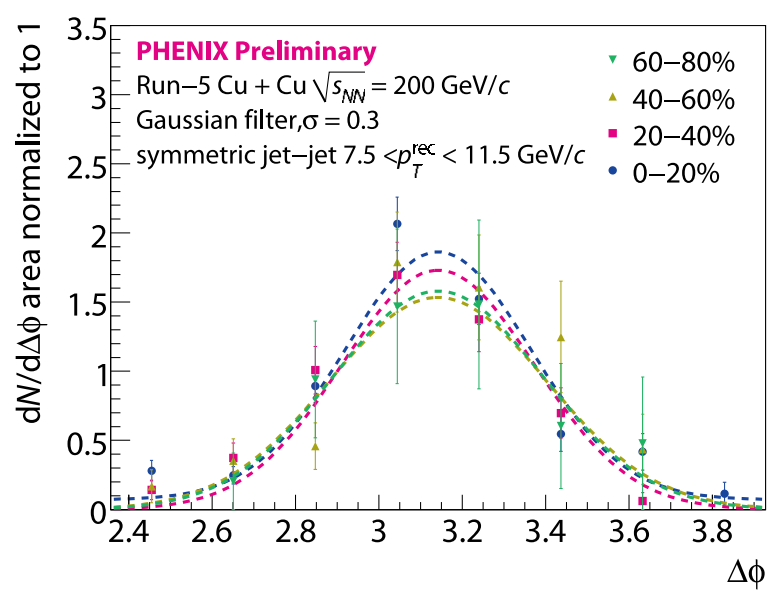

Figure 11. Azimuthal jet-jet correlation measured by the PHENIX experiment in $\mathrm{Run}-5 \mathrm{Cu}+\mathrm{Cu}$ collisions at $\sqrt{s_{N N}}=200 \mathrm{GeV}$ for jets with $7.5 \mathrm{GeV} / c<p_{T}<11.5 \mathrm{GeV} / c$ and several centrality classes (see legend). Figure taken from [42].

\section{5 -hadron correlations}

In contrast to hadrons, direct photons do not interact via the strong force, escape the nuclear matter unmodified (cf. Figure 1) and can thus be used as a calibrated probe of the energy of an initial hard scattering. Therefore are direct photons often considered as a "golden" probe of hot and dense nuclear matter. At leading order, direct photons are produced via QCD "Compton" scattering, $q+g \rightarrow q+\gamma$. In the limit of vanishing initial transverse momentum, the photon and the final state quark are emitted back-to-back in azimuth and the photon exactly balances the transverse momentum of the quark jet. We note that this balance is however only approximate because the non-zero transverse momentum of the colliding partons inside nucleons $\left(k_{T}\right)$ translates to imbalance and acoplanarity between the photon and the quark jet.

To measure fragmentation function of the quark jet, measurements of direct photon-hadron correlations were explored by both PHENIX [45] and STAR [46] experiments. For brevity, we concentrate here only on recent results from the PHENIX experiment. The measured momentum of the direct photon $\left(p_{T}^{\gamma}\right)$ is very good approximation of the initial jet $p_{T}$ and in turn the ratio $z_{T}=p_{T}^{h} / p_{T}^{\gamma}$ can be used to a good approximation for the jet fragmentation function measurements. To extract the direct photon-hadron yields, in $\mathrm{p}+\mathrm{p}$ collisions a photon isolation cut was applied and in $\mathrm{Au}+\mathrm{Au}$ collisions a statistical subtraction had to be used instead due to large background. To assess medium modification of the jet fragmentation function, a nuclear modification factor of the correlated yield, $I_{\mathrm{AA}}$, was then calculated. As the medium modification of fragmentation function is expected to be more pronounced at low $z_{T}$ values, the fragmentation functions are commonly plotted as a function of the variable $\xi=\ln \left(1 / z_{T}\right)$. The fully corrected $I_{\mathrm{AA}}$ is shown in the left panel of Figure 12. In absence of medium modification, $I_{\mathrm{AA}}$ should be unity, however the data indicate a clear suppression at low $\xi$ compensated by enhancement at higher $\xi$. Taking into account all systematic uncertainties, the probability that $I_{\mathrm{AA}}$ is consistent with unity is less than $0.1 \%$ for $\xi>0.8$. The data are also compared with two models, BW-MLLA [47] and YaJEM-DE [48]. Both models are in qualitative agreement with the data and reproduce suppression at low $\xi$ due to parton energy loss in $\mathrm{Au}+\mathrm{Au}$ collisions as well as the 

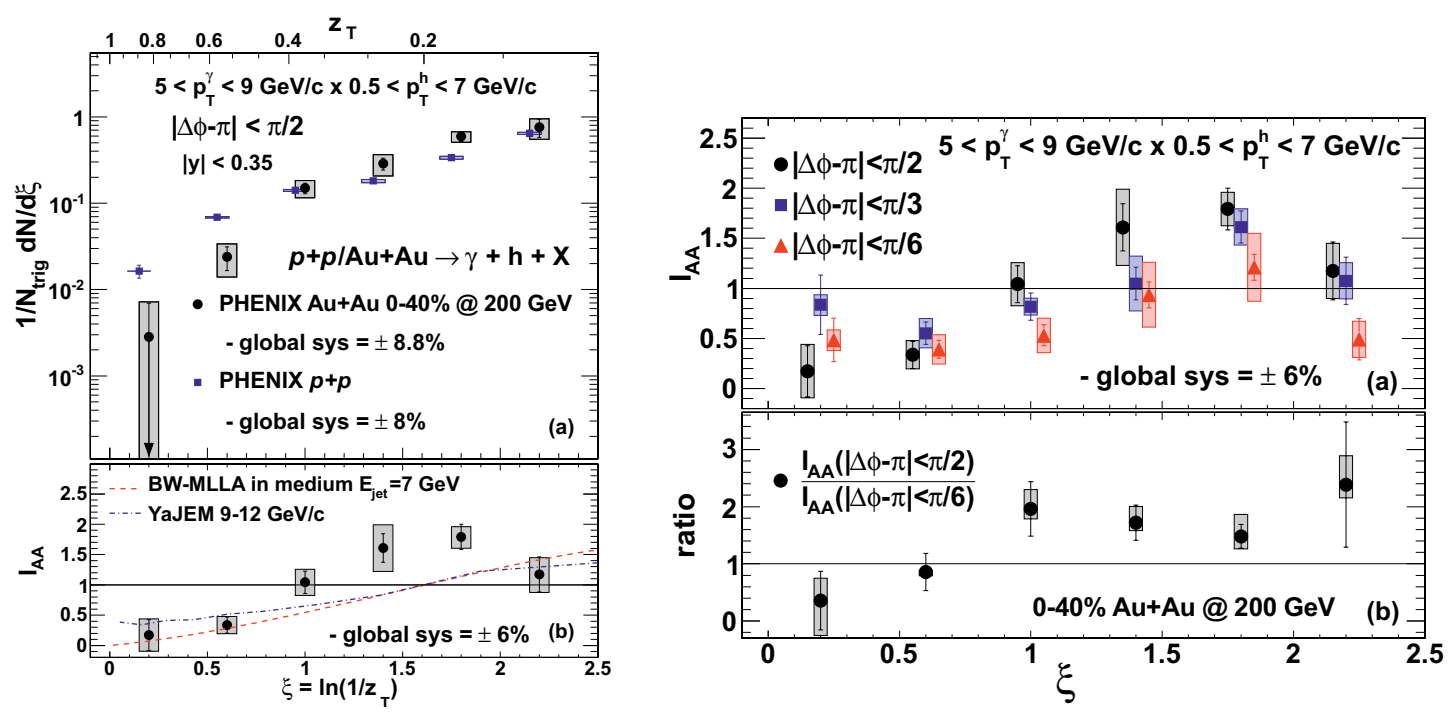

Figure 12. (Left) Per trigger yield as a function of $\xi$ for $p+p$ collisions (squares) and $0-40 \%$ most central $\mathrm{Au}+\mathrm{Au}$ collisions (circles). For reference, the dependence on $z_{T}$ is also indicated at top of the y-axis. The bottom panel shows nuclear modification factor $I_{\mathrm{AA}}$ of fragmentation functions together with predictions from BW-MLLA [47] (dashed line) and YaJEM-DE [48] (dot-dashed curve). (Right) The top panel shows $I_{\mathrm{AA}}$ for the full away-side $(|\Delta \phi-\pi|<\pi / 2)$ (circles) and for two restricted away-side integration ranges, $|\Delta \phi-\pi|<\pi / 3$ (squares) and $|\Delta \phi-\pi|<\pi / 6$ (triangles). The bottom panel shows the ratio of the $I_{\mathrm{AA}}$ for $|\Delta \phi-\pi|<\pi / 2$ to $|\Delta \phi-\pi|<\pi / 6$.

increase of $I_{\mathrm{AA}}$ with $\xi$ due to the redistribution of the lost energy into particles with lower momenta. However, neither of the two models is able to reproduce details of the transition from suppression to enhancement.

In order to shed light on the angular redistribution of the lost energy, PHENIX studied $I_{\mathrm{AA}}$ in three different azimuthal ranges. The results are depicted in the right panel of Figure 12. Reducing the integration range from $|\Delta \phi-\pi|<\pi / 2$ results in lower enhancement and shifts the transition point to higher $\xi$. For the azimuthal range $|\Delta \phi-\pi|<\pi / 6$, the enhancement for $\xi>1.0$ is found negligible, while significant suppression for $\xi<0.8$ still persists. In the bottom panel, the ratio of $I_{\mathrm{AA}}$ in the full away-side integration range to that in the $|\Delta \phi-\pi|<\pi / 6$ case is plotted. This double ratio demonstrates a significant variation in observed $I_{\mathrm{AA}}$ as a function of the integration range and clearly points to the fact that the soft particles are preferentially emitted at large angles.

\section{Summary and outlook}

The amount of results achieved by the STAR and PHENIX experiments at RHIC is truly impressive. In this proceedings, only a selection of recent results on hard probes could be featured. Hard probes have proven to be very useful tool to study properties of hot and dense matter created in heavyion collisions at RHIC energy regime. The mechanism of energy loss of heavy quarks in hot and dense medium is not yet fully understood and intense theoretical discussions about the importance of radiative and collisional energy loss and other in-medium effects are ongoing. Studies of fully reconstructed jets have proven to be possible even at the RHIC energy regime, where their production 
rate is very small. Both STAR and PHENIX experiments have achieved recently great steps forward in these challenging studies.

The experimental study of the hot and dense nuclear matter entered a new era with the operation of the Large Hadron Collider (LHC) at CERN, which enables to study nuclear matter at even higher energies and temperatures than achievable at RHIC. Nonethless, studies of the hot and dense QCD matter in the RHIC energy regime will remain to play a key role in the field of heavy-ion physics even in the LHC era. The RHIC facility is unique and enables to investigate a large variety of colliding systems over a broad range of collision energies. Both STAR and PHENIX experiments will from 2014 run with greatly enhanced capabilities allowing among others studies of heavy quark production via direct reconstruction of secondary vertices of charm and beauty hadron decays. This together with the RHIC-II luminosity upgrade will enable STAR and PHENIX to enter a new era of precision and comprehensive measurements.

\section{Acknowledgements}

The work has been supported by the grant 13-02841S of the Czech Science Foundation (GACR).

\section{References}

[1] J. Adams et al. (STAR Collaboration), Nucl.Phys. A757, 102 (2005), nucl-ex/0501009

[2] K. Adcox et al. (PHENIX Collaboration), Nucl.Phys. A757, 184 (2005), nucl-ex/0410003

[3] B. Back, M. Baker, M. Ballintijn, D. Barton, B. Becker et al., Nucl.Phys. A757, 28 (2005), nucl-ex/0410022

[4] I. Arsene et al. (BRAHMS Collaboration), Nucl.Phys. A757, 1 (2005), nucl-ex/0410020

[5] M.L. Miller, K. Reygers, S.J. Sanders, P. Steinberg, Ann.Rev.Nucl.Part.Sci. 57, 205 (2007), nucl-ex/0701025

[6] S. Horvat (STAR), PoS CPOD2013, 002 (2013)

[7] S.P. Horvat (STAR), J.Phys.Conf.Ser. 446, 012017 (2013), 1303.7260

[8] M. Gyulassy, X.N. Wang, Comput.Phys.Commun. 83, 307 (1994), nucl-th/9502021

[9] B. Zhang, C. Ko, B.A. Li, Z.w. Lin, Phys.Rev. C61, 067901 (2000), nucl-th/9907017

[10] E. Sangaline (STAR Collaboration), Nucl.Phys. A904-905, 771c (2013)

[11] B. Abelev et al. (STAR Collaboration), Phys.Lett. B655, 104 (2007), nucl-ex/0703040

[12] L. Adamczyk et al. (STAR Collaboration), Phys.Rev. D86, 072013 (2012), 1204.4244

[13] D. Tlusty (STAR collaboration), Nucl.Phys. A904-905, 639c (2013), 1211. 5995

[14] M. Cacciari, P. Nason, R. Vogt, Phys.Rev.Lett. 95, 122001 (2005), hep-ph/0502203

[15] S. Adler et al. (PHENIX Collaboration), Phys.Rev.Lett. 96, 032001 (2006), hep-ex/0508034

[16] A. Adare et al. (PHENIX Collaboration), Phys.Rev.Lett. 97, 252002 (2006), hep-ex/0609010

[17] H. Agakishiev et al. (STAR Collaboration), Phys.Rev. D83, 052006 (2011), 1102 . 2611

[18] Y.L. Dokshitzer, D. Kharzeev, Phys.Lett. B519, 199 (2001), hep-ph/0106202

[19] S. Adler et al. (PHENIX Collaboration), Phys.Rev.Lett. 94, 082301 (2005), nucl-ex/0409028

[20] S. Adler et al. (PHENIX Collaboration), Phys.Rev.Lett. 96, 032301 (2006), nucl-ex/0510047

[21] B. Abelev et al. (STAR Collaboration), Phys.Rev.Lett. 98, 192301 (2007), nucl-ex/0607012

[22] B.W. Zhang, E. Wang, X.N. Wang, Phys.Rev.Lett. 93, 072301 (2004), nucl-th/0309040

[23] S. Wicks, W. Horowitz, M. Djordjevic, M. Gyulassy, Nucl.Phys. A784, 426 (2007), nucl-th/0512076 
[24] A. Adil, I. Vitev, Phys.Lett. B649, 139 (2007), hep-ph/0611109

[25] W. Xie (STAR Collaboration), Nucl.Phys. A904-905, 170c (2013)

[26] M. He, R.J. Fries, R. Rapp, Phys.Rev.Lett. 110, 112301 (2013), 1204.4442

[27] P.B. Gossiaux, J. Aichelin, M. Bluhm, T. Gousset, M. Nahrgang et al., PoS QNP2012, 160 (2012), 1207.5445

[28] A. Adare et al. (PHENIX Collaboration) (2013), 1310.8286

[29] A. Adare et al. (PHENIX Collaboration), Phys.Rev. C88, 024906 (2013), 1304.3410

[30] J. Cronin, H.J. Frisch, M. Shochet, J. Boymond, R. Mermod et al., Phys.Rev. D11, 3105 (1975)

[31] T. Renk, K. Eskola, Phys.Rev. C75, 054910 (2007), hep-ph/0610059

[32] J. Putschke (STAR Collaboration), Eur.Phys.J. C61, 629 (2009), 0809. 1419

[33] S. Salur (STAR Collaboration), Eur.Phys.J. C61, 761 (2009), 0809. 1609

[34] M. Ploskon (STAR Collaboration), Nucl.Phys. A830, 255C (2009), 0908 . 1799

[35] E. Bruna (STAR Collaboration), Nucl.Phys. A830, 267C (2009), 0907.4788

[36] M. Cacciari, G.P. Salam, G. Soyez, Eur.Phys.J. C72, 1896 (2012), 1111.6097

[37] W. Vogelsang, private communication with the STAR collaboration (2009)

[38] I. Vitev, B.W. Zhang, Phys.Rev.Lett. 104, 132001 (2010), 0910 . 1090

[39] J. Rusnak (STAR Collaboration), Hard Probes 2013, Inclusive Spectrum of Fully Reconstructed Charged Jets in Central $\mathrm{Au}+\mathrm{Au}$ Collisions at $\sqrt{s_{N N}}=200 \mathrm{GeV}$ by the STAR Collaboration (2013)

[40] Y.S. Lai, B.A. Cole (2008), 0806.1499

[41] Y.S. Lai (PHENIX collaboration) (2009), 0911. 3399

[42] Y.S. Lai (PHENIX Collaboration), Nucl.Phys. A830, 251C (2009), 0907 . 4725

[43] Y.S. Lai (PHENIX Collaboration), Nucl.Phys. A855, 295 (2011)

[44] A. Adare et al. (PHENIX Collaboration), Phys.Rev.Lett. 101, 162301 (2008), 0801.4555

[45] A. Adare et al. (PHENIX Collaboration), Phys.Rev.Lett. 111, 032301 (2013), 1212.3323

[46] B. Abelev et al. (STAR Collaboration), Phys.Rev. C82, 034909 (2010), 0912 . 1871

[47] N. Borghini, U.A. Wiedemann (2005), hep-ph/0506218

[48] T. Renk, Phys.Rev. C84, 067902 (2011), 1110. 2313 